\title{
Towards Green 3D-Microfabrication of Bio-MEMS Devices Using ADEX Dry Film Photoresists
}

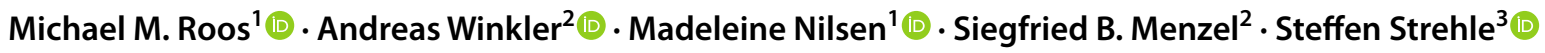

Received: 15 July 2020 / Revised: 29 May 2021 / Accepted: 7 June 2021 / Published online: 3 September 2021

(c) The Author(s) 2021

\begin{abstract}
Current trends in miniaturized diagnostics indicate an increasing demand for large quantities of mobile devices for health monitoring and point-of-care diagnostics. This comes along with a need for rapid but preferably also green microfabrication. Dry film photoresists (DFPs) promise low-cost and greener microfabrication and can partly or fully replace conventional silicon-technologies being associated with high-energy demands and the intense use of toxic and climate-active chemicals. Due to their mechanical stability and superior film thickness homogeneity, DFPs outperform conventional spin-on photoresists, such as SU-8, especially when three-dimensional architectures are required for micro-analytical devices (e.g. microfluidics). In this study, we utilize the commercial epoxy-based DFP ADEX to demonstrate various application scenarios ranging from the direct modification of microcantilever beams via the assembly of microfluidic channels to lamination-free patterning of DFPs, which employs the DFP directly as a substrate material. Finally, kinked, bottom-up grown silicon nanowires were integrated in this manner as prospective ion-sensitive field-effect transistors in a bio-probe architecture directly on ADEX substrates. Hence, we have developed the required set of microfabrication protocols for such an assembly comprising metal thin film deposition, direct burn-in of lithography alignment markers, and polymer patterning on top of the DFP.
\end{abstract}

Keywords Dry film photoresist $\cdot$ Lithography $\cdot 3 \mathrm{D}$ Microfabrication $\cdot$ Microcantilevers $\cdot$ Lab-on-a-chip $\cdot$ Microprobes

$\begin{array}{ll}\text { Abbreviations } \\ \text { AFM } & \text { Atomic force microscope } \\ \mathrm{CAD} & \text { Computer-aided design } \\ \mathrm{CD} & \text { Critical dimension } \\ \mathrm{DFP} & \text { Dry film photoresist } \\ \mathrm{MEMS} & \text { Micro electro mechanical systems } \\ \mathrm{NEMS} & \text { Nano electro mechanical systems } \\ \mathrm{PET} & \text { Polyethylene terephthalate } \\ \mathrm{PGMEA} & \text { Propylene glycol methyl ether acetate } \\ \mathrm{PP} & \text { Polypropylene } \\ \mathrm{SiO}_{2} & \text { Silicon oxide } \\ \mathrm{SF}_{6} & \text { Sulfur hexafluoride } \\ \mathrm{UV} & \text { Ultraviolet }\end{array}$

Steffen Strehle

steffen.strehle@tu-ilmenau.de

1 Institute of Electronic Devices and Circuits, Ulm University, Albert-Einstein-Allee 45, 89081 Ulm, Germany

2 Group Acoustic Microsystems, IFW Dresden, SAWLab Saxony, Helmholtzstraße 20, 01069 Dresden, Germany

3 Institute of Micro- and Nanotechnologies MacroNano®, Microsystems Technology Group, Technische Universität Ilmenau, Max-Planck-Ring 12, 98693 Ilmenau, Germany

\section{Introduction}

Miniature analytical devices represent a crucial component in the preparation of future personalized medicine with emphasis to point-of-care microscale total analysis systems ( $\mu$ TAS). Such devices enable, for instance, virus detection, personalized cancer diagnostics, disease biomarker registration as well as single cell and cell culture studies [1-3]. Thus, different types of miniature bioanalytical devices for a multitude of application cases were proposed and demonstrated. Microfluidic devices operate mainly with liquid samples and were discussed for the sorting, analysis and manipulation of single-cells by electric, mechanical, biochemical, and electrophoretic methods, by means of piezoactuators and optical and acoustic tweezers [4]. Hence, they enabled even to study the role of mechanical properties and biochemical signals on the migration and invasion of metastasizing cancer cells [5]. In contrast to 'bulky' microfluidic chips, microscale probes are required for minimalinvasive in-vitro and in-vivo studies of living cells $[6,7]$. For instance, high resolution detection of the transepithelial transport of $\mathrm{K}^{+}-\mathrm{Na}^{+}$-, and $\mathrm{Cl}^{-}$-ions in the thin lining fluid at the surface of pulmonary epithelial cells would elucidate 
the underlying processes of diseases like cystic fibrosis and lung oedema [8,9]. Miniature solid-state ion-selective electrodes were demonstrated for in-vivo measurements of the $\mathrm{K}^{+}$-concentration in rodent brains [7], but were built on silicon. Lieber et al. presented multiple strategies to integrate single silicon nanowire field-effect transistors as biological signal transducers and employed polymeric probes (SU-8 resist, Microchem Corp.) on a silicon substrate and other probe designs to record the heartbeat of single cardiomyocite cells in-vitro [10-12]. Syringe injectable SU-8-based mesh electronics were recently proposed for therapeutic applications, demonstrating in-vivo recording of physiological parameters in rodent brains [13-15].

As these examples demonstrate, elaborated 3D microfabrication tools are essential to realize 3D microscale features, such as microfluidic channels as well as integrated microscale sensors and actors. For the implementation of microfabricated devices on an industrial scale, manufacturing processes must, furthermore, meet high requirements comprising bio-device reliability, hygiene aspects, safety, and scalability. Due to biological safety and medical hygiene, many of the aforementioned miniature devices must be designed for single-use only, which is already true for many of today's macroscopic medical devices and device parts [16]. In this regard, but also in a more general point of view, green fabrication strategies should be supported and developed also in the field of microsystems, which includes the reduction of the number of required process steps, the total amount of waste, the total energy consumption, and the overall need for toxic and environmentally harmful substances.

Here, the use of the commercial epoxy-based dry film photoresist (DFP) ADEX ${ }^{\mathrm{TM}}$ (DJ MicroLaminates, Inc.) is discussed as a promising material for microdevice fabrication. DFPs are solid photosensitive polymeric foils, originally developed for the production of printed circuit boards. They can be directly laminated onto wafers, chips, circuit boards, and other substrates and micropatterned by ultraviolet (UV) optical lithography, providing a promising alternative to conventional spin-on photoresists. With their merely organic chemicals, DFPs can be considered environmentfriendly in comparison to the conventional silicon- and also glass-based microfabrication processes. The latter comprise commonly energy-intensive single-crystalline silicon synthesis and wafer fabrication as well as aggressive, toxic and environmentally harmful microtechnological process chemicals, such as the highly toxic hydrogen fluoride or the climate-active sulfur hexafluoride $\left(\mathrm{SF}_{6}\right)$ [17]. One kilogram of $\mathrm{SF}_{6}$ can be potentially more than 20.000 times as climateactive as $1 \mathrm{~kg}$ of $\mathrm{CO}_{2}$ with regard to a reference period of 100 years [18]. Hence, such process should be avoided if possible.

Based on this, polymeric materials, which comprise also biopolymers like chitin [19], lignin [20], shellac [21] and silk [22], are in general appealing and might be preferred for larger scale fabrication in future wherever possible as a cheaper and more environment-friendly alternative [23]. Nevertheless, SU-8-based microfabrication and the widespread soft-lithography methods, based e.g. on stamping, moulding, and nano-imprinting, exist as well but require solid substrates, which are mainly silicon or glass wafers. Additionally, 3D-printing and two-photon lithography are highly versatile tools for the creation of 3D-polymeric structures but they are currently only suited for prototyping rather than for large scale fabrication of identical devices [23-25]. Conventional lamination-based techniques allow in principle 3D-manufacturing based on patterned multilayer-architectures. However, they are based on stacking and bonding of multiple previously laser- or knife-cut layers [24]. This is hardly compatible with high-throughput manufacturing at microscale resolution and poses also the challenge of accurate cutting and multilayer alignment at each lamination layer [24]. Another approach for lithographic 3D microfabrication is "inclined UV lithography" or "multidirectional UV lithography" [26] that could be used alternatively or complementary to multilayer patterning but requires suitable lithography tools.

In contrast to these issues, DFPs show several general advantages for the manufacturing of 3D-microstructures, biomedical systems, or in general for MEMS that make them superior if compared to conventional spin-on photoresists, such as the epoxy-based photoresist SU-8, namely:

- Defined and homogeneous film thickness and material properties over large areas as well as high planarity

- Possibility to deposit thick films (e.g. commercial epoxybased DFP SUEX ${ }^{\circledR}$ (DJ MicroLaminates, Inc.): up to $1000 \mu \mathrm{m})$

- Low outgassing of potentially harmful solvents

- No soft-bake required

- Relatively low costs (naturally depending on the overall system design)

- Overall facile and rapid processing

- High suitability for multilayer assembly

The unique possibilities for microsystems assembly were already shown for some applications, using self-made and commercial DFPs, and comprise microfluidic channels [27, 28], MEMS packaging, switches [29], sandblasting and etch masks [30], and electroplating moulds [31]. Johnson et al. and Lemke et al. achieved high aspect ratios of up to 40 using SUEX DFP [32, 33] and up to 100 using the commercial DFP mr-X (MRT GmbH)[34]. Structures made of the acrylic DFP product Ordyl SY (Elga Europe s.r.l) were used as spacers, bonding materials, and as lithography alignment features within a complex fluidic valve that involved two layers of wafers and a Peltier cooler [35]. Wangler et al. 
[36] investigated the effect of lamination temperature, roller speed, and pressure in a double-layer lamination process for the commercially available, hydrophilic, and epoxy-based DFP TMMF (Tokyo Ohka Kogyo Co. Ltd.); and they created ideally covered microchannel of up to $2 \mathrm{~mm}$ self-supporting width. Other groups studied multilayer lithography of the commercial epoxy-based DFPs DF-1000 (EMS Nagase Group) or PerMX (Dupont) combined with conventional SU-8 [37, 38] and assembled for instance a unique fluidic "coil" with three separated fluids [38]. Finally, also microarray print heads involving multilayer TMMF DFP lithography were shown with the remark that DFPs can drastically reduce the costs and lead time of MEMS production.

Despite their versatile use, various DFPs have already disappeared from the market comprising PerMX, Shipley 5038 (Dow Chemical Company), Etertec HT/HQ (Eternal Chemical Co. Ltd.), ME1050 (Hitachi Chemical Co. Ltd.) or mr-X. However, six different DFPs are currently commercially available as listed in the appendix. These commercial products differ significantly with respect to their original material base (e.g. acrylic or epoxy), the handling, the available film thickness range, the optical properties, and the selling price.

One major drawback of DFPs, compared to the vastly exploited spin-on epoxy resist SU-8 (Microchem Corp.), is the limited experience and knowledge of the overall reproducibility, long-term effects as well as the stability of technological process parameters, which is required to make this technology ready for a broader industrial use [39]. Suspended DFPs within multilayer stacks are prone to so-called sagging based on the geometrical design and the DFP properties. This issue is well known from other techniques like soft nano-imprint lithography [40]. Hence, a proper design and optimization of the process parameters (i.e. temperature, pressure and speed) with respect to the intended design is required.

To facilitate the usage of DFPs, we discuss here possibilities of a so far hardly investigated material system, namely the epoxy-based DFP ADEX. We present several microfabrication approaches that, compared to previously shown SU-8 based strategies [11], allow to access further application scenarios or that are significantly more facile than previous strategies. We will discuss them by increasing importance of the DFP within the device fabrication strategy. Hence, we present at first a strategy (previously published in [41]) for the direct microtechnological modification of freestanding silicon nitride cantilever beams, which are frequently used for (bio-)AFM [42] and for microcantilever assisted biosensors [43]. DFP ADEX is used in this case as a photoresist material. Second, we demonstrate the use of DFP ADEX for the manufacturing of microfluidic channel walls and lids. Previously required process steps, such as gluing, thermal fusion bonding, or ultrasonic welding of a lid onto the top of the channel wall become obsolete. Last, we introduce a lamination-free implementation of DFPs as substrates themselves that allows patterning of DFP ADEX via direct laser lithography, which is beneficial for prospective polymeric lab-on-chip, microprobe, and mesh electronic devices. We show and discuss furthermore in this context, the creation of metal and polymeric structures on top of ADEX as well as the direct burn-in of alignment markers to support multilayer processes. In summary of these techniques, we demonstrate the integration of bottom-up grown kinked silicon nanowires in a frequently discussed biosensor field-effect transistor configuration $[10,11,44]$ on top of DFP ADEX. Making several previously required process steps and materials obsolete, our DFP strategy enhances the overall fabrication efficiency, while lowering the overall environmental impact. Handling of SUEX and ADEX DFPs was observed to differ slightly. Due to SUEXs brittleness at room temperature, the so-called thick film sheets (thickness $>100 \mu \mathrm{m}$ ) were more difficult to handle than the flexible ADEX thin films (thickness $\leq 75 \mu \mathrm{m}$ ). Therefore, we focus here on ADEX rather than SUEX. So-called SUEX thin dry film sheets that appeared recently on the market, were hardly included in this study.

\section{Materials and Methods}

DFPs ADEX and SUEX were bought from DJ MicroLaminates Corporation. All process chemicals were purchased from MicroChemicals GmbH.

Direct lamination of ADEX films onto the substrates was performed with a hot roll laminator (LMG Sky 335 R6) after the removal of the polypropylene (PP) bottom cover film using $60-65^{\circ} \mathrm{C}$ at the minimal velocity of ca. $6 \mathrm{~mm} / \mathrm{s}$. The mechanical lamination pressure could not be controlled. To achieve an overall uniform coating and to avoid bubble formation, an additional bake at $65^{\circ} \mathrm{C}$ for 5 min was added.

If not specified otherwise, lithographic exposure was performed by a UV laser lithography tool ( $\mu$ PG101, Heidelberg Instruments, $375 \mathrm{~nm}$, writing speed $5 \mathrm{~mm}^{2} / \mathrm{min}$, focal length $4 \mathrm{~mm}$ ) after removal of the top protective liner. After exposure, a post-exposure bake was done at $95{ }^{\circ} \mathrm{C}$ for 5-10 $\mathrm{min}$ on a hotplate. Finally, the resist was developed either in propylene glycol methyl ether acetate (PGMEA) or in cyclohexanone according to the manufacturer specifications with development times ranging from 2 to $20 \mathrm{~min}$ depending on the utilized resist thickness. Detailed studies of the fabrication parameters for lamination-free implementation of ADEX and SUEX are attached in the supplementary materials, Sect. 3.

Photoresist AZ 5214 E (used in positive tone) was spincoated at $4000 \mathrm{rpm}$ for $1 \mathrm{~min}$, dried at ambient conditions 
for $4 \mathrm{~h}$, exposed at $0.65 \mathrm{~mW}$ effective laser power, and developed with the alkaline developer AZ MIF-726. Photoresist SU-8 (2000.5) was spin-coated at 2000 rpm, dried overnight under ambient conditions, exposed at a laser power of $1.3 \mathrm{~mW}$, post-exposure baked at $95^{\circ} \mathrm{C}$, and developed in cyclohexanone for $1 \mathrm{~min}$.

Gold metal structures were deposited with a Leybold L560 thermal evaporator $(5 \mathrm{~nm} \mathrm{Ti}+100 \mathrm{~nm} \mathrm{Au})$ with a thin layer of titanium for improved adhesion on ADEX. Transmission spectra were measured with a dispersive spectrophotometer (Shimadzu UV-3101PC, controlled by UVProbe 2.33 software package). The spectrometer had a resolution of $0.1 \mathrm{~nm}$. The combination of deuterium (D2) and the tungsten-halogen (WI) lamps enabled a spectral range between 190 and $800 \mathrm{~nm}$.

\section{Results and Discussion}

One major limitation of conventional spin-on resists, such as SU-8, is their restriction to mainly planar surfaces if a homogeneous resist coverage shall be achieved. At edges or on 3D features, but also on larger planar surfaces, the resist thickness tends to be inhomogeneous, making it difficult to ensure a reliable and reproducible fabrication quality. With regard to this, DFPs provide the desirable feature that they can be laminated onto a substrate. Thus, homogeneous coatings across large areas and 3D patterned surfaces are possible. Another significant advantage of laminated DFPs, compared to spin-on resists, is the elimination of the so-called softbake processing step, which is known to frequently cause the formation of resist cracks.

The schematic illustration in Fig. 1a depicts the conventional implementation of DFPs as a single layer photoresist on a planar substrate. DFPs are laminated onto the substrate, locally exposed by UV light using a lithographic mask or laser lithography, and finally, the DFP is developed. This strategy for microfabrication of planar patterns allows reaching high aspect ratios up to at least 15 , as the $75 \mu \mathrm{m}$ thick lamellar structures in Fig. 1b demonstrate.

Instead of crack formation during the softbake, DFPs can suffer from bubble formation at the interface between the DFP and the substrate due to air trapping occurring during the lamination process. Bubble-free DFP coatings are achievable, partly supported by the aforementioned additional bake at $65^{\circ} \mathrm{C}$ for $5 \mathrm{~min}$, but the risk for bubble trapping increases in particular at edges within a ca. $500 \mu \mathrm{m}$ wide surrounding region (Fig. 1c) and obviously for spacious laminations simply due to statistics. This issue must be addressed in future for instance by developing and employing more advanced lamination devices.

As a certain light absorption is required to activate the photoinitiators and to expose the photoresist, the
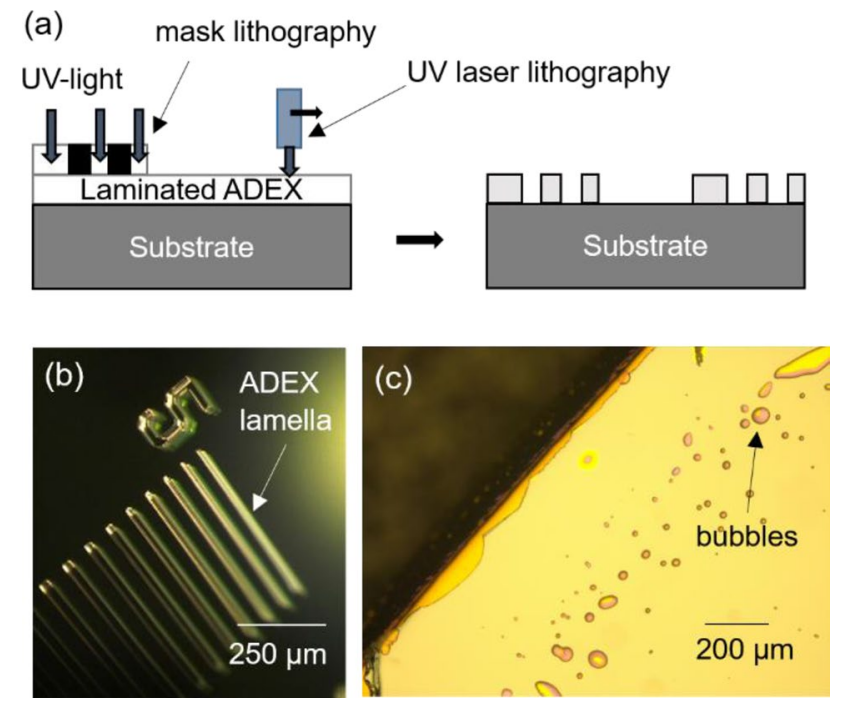

Fig. 1 DFPs as a photoresist: a Scheme of mask photolithography and laser lithography on laminated DFPs analogue to microfabrication with SU-8. b Lamellar structure of varied thickness (ADEX: height of the lamella $75 \mu \mathrm{m}$, minimal thickness $5 \mu \mathrm{m}$ equivalent to an aspect ratio of 15). c Inclusion of bubbles between ADEX and the coated wafer during lamination

transmission spectra of an unexposed resist can give valuable insights into the lithographically usable optical wavelength range to expand the overall DFP usability and to enable multi-wavelength and grayscale exposure $[45,46]$. Hence, we measured the transmission spectra of two non-exposed ADEX foils of $20 \mu \mathrm{m}$ and $50 \mu \mathrm{m}$ thickness, laminated on amorphous silicon oxide $\left(\mathrm{SiO}_{2}\right)$ chips, using a dispersive spectrophotometer. Figure 2 shows the transmission spectra and the extinction coefficient for the wavelength range that is commonly used for optical lithography. Based on these measurements, DFP ADEX can be structured within the wavelength range from violet to ultraviolet (340-425 nm). Above a wavelength of about $450 \mathrm{~nm}$, the unexposed resist is almost transparent with a transmission of larger than $96 \%$. Compared to SU-8, ADEX shows a slightly lower optical transmission, but an overall similar spectral behavior [45].

According to Beer-Lambert's law of attenuation of light through a medium at negligible reflection, the optical intensity in the medium decays exponentially (see Fig. 2, inset). This results in a reduction of the optical dose with increasing distance from the resist surface. Therefore, higher lithography wavelengths, e.g. the h-line $(405 \mathrm{~nm})$, are expected to be suited to fully expose ADEX-layers up to mm thickness and make ADEX DFP also well-compatible with conventional mask-based optical lithography.

The benefit from using DFPs becomes apparent, when precise polymeric structures shall be fabricated on 3D patterned or even freestanding substrates. In a first study (see [41] for more details), we demonstrated that DFPs can be 


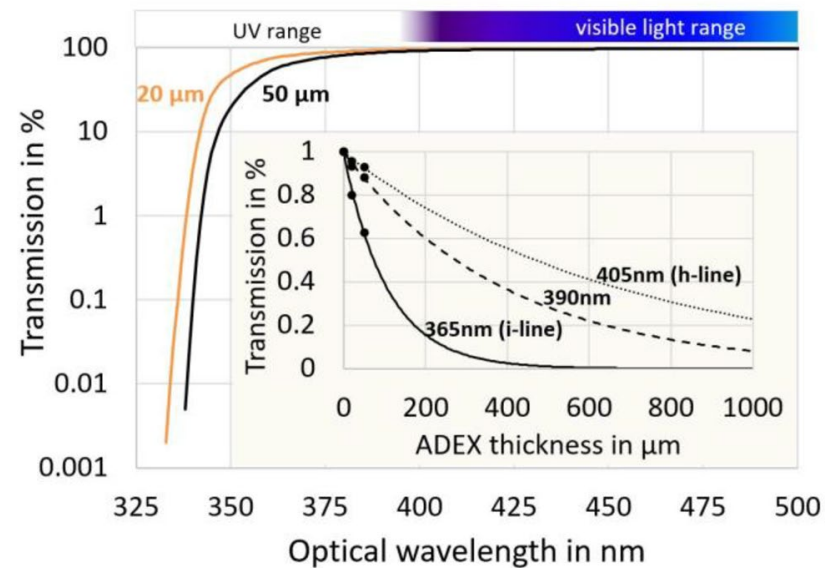

Fig. 2 Optical transmission spectra of two non-exposed ADEX foils with different thickness $(20$ and $50 \mu \mathrm{m})$, and inset showing the extrapolated optical transmission (exponential trends based on indicated measurement points according to Beer-Lambert's law) for very thick ADEX foils at the most important optical wavelengths

directly laminated onto freestanding silicon nitride microcantilevers yielding a homogeneous resist coating that is not possible with conventional spin-coating of liquid resists. This enabled the direct lithographic patterning of microcantilever beams. Figure 3 shows some examples of silicon-nitride microcantilever beams that were modified by DFP-lithography. To demonstrate the versatility of this technique, metal structures were created by lift-off (Fig. 3a), holes were created by dry etching (Fig. 3b), and polymeric pillars were made directly from DFPs (Fig. 3c). Such DFP pillar probes were already successfully used within AFM to investigate the elastic modulus of soft biological cells that could otherwise be damaged by conventional pyramidal-shaped cantilever tips [41]. Notably, even commercial microcantilevers already equipped with an AFM scanning tip could be successfully modified in this manner without any recognizable deterioration of the scanning tip. This
DFP process is facile and is merely based on lamination and lithography, and therefore, well transferable to the industrial scale. Compared to an alternative resist deposition by socalled spray coating, direct and localized lamination of the DFP at exactly the required area minimizes, furthermore, the need for polymeric resources and the amount of waste. As we showed recently, microcantilevers can be also fabricated from DFP ADEX [47].

\subsection{Multilayer DFPs for Microfluidic Channels}

As demonstrated, DFPs can be readily patterned via scalable optical lithography. The use of DFPs is, furthermore, intrinsically not limited to single-layer patterning. Sequential lamination and lithographic patterning of multiple DFP layers (see scheme in Fig. 4a) enables in principle to create microfluidic channels as shown before [35]. Notably, DFP methods for microfluidic channel assembly seem still to be rather unknown to the microfluidic community. Changing this would foster the continuation of the rapid spread of microfluidics [24]. At this point one must admit that still further research is required aside from pure manufacturing aspects to study physical, chemical, and biological properties further to optimize them for improved quality and reliability.

Figure $4 b, c, d$ show some examples that demonstrate ADEX structures for the assembly of microfluidic channels. For the fabrication of microfluidic channels (Fig. 4b), the $37.5 \mu \mathrm{m}$ thick channel walls were created at first from a $75 \mu \mathrm{m}$ thick DFP that was laminated onto the substrate (about $6 \mathrm{~mm} / \mathrm{s}, 65^{\circ} \mathrm{C}$ ), exposed via laser lithography ( $2 \times$ exposed at $\left.0.25 \mathrm{~W}, 1.25 \mathrm{~mm}^{2} / \mathrm{min}\right)$, post-exposure baked, and developed (20 min in cyclohexanone). Subsequently, the $5 \mu \mathrm{m}$ thick lid structures were fabricated in an analogous manner without any adjustments with regard to the DFP-thickness (details in Sect. 2). In order to emphasize the versatility of this fabrication approach, $5 \mu \mathrm{m}$ thick roof
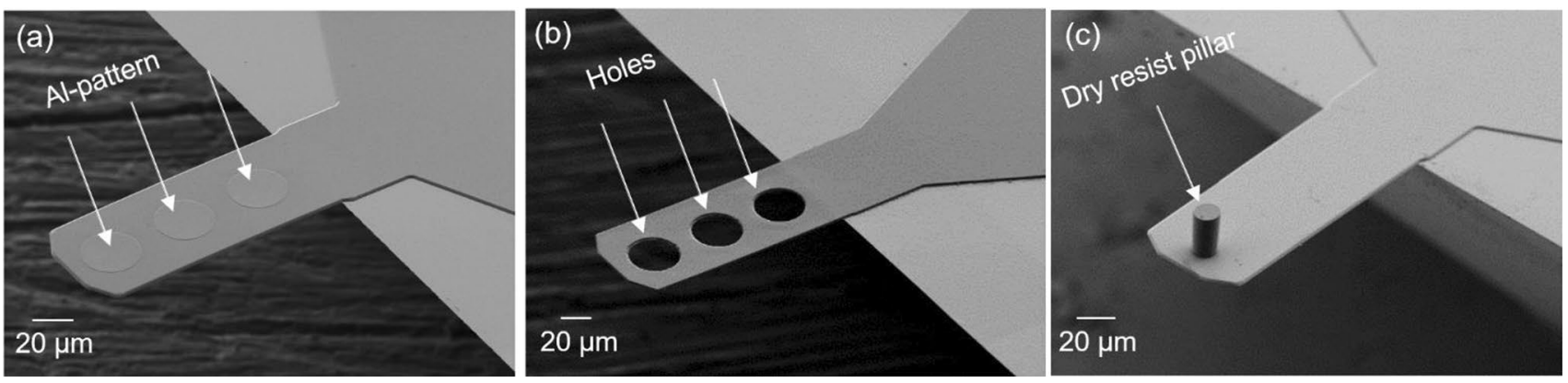

Fig. 3 Implementation of DFPs in the field of AFM-cantilevers: Structural modification of silicon nitride cantilevers (length: $150 \mu \mathrm{m}$, width: $65 \mu \mathrm{m}$ ) implementing $5 \mu \mathrm{m}$ thick laminated DFP ADEX: a as a resist for manufacturing metal structures in a liftoff-process, $\mathbf{b}$ as an etching mask for RIE-etching of unexposed circles, and $\mathbf{c}$ as a material for making high-aspect-ratio polymeric structures (height: $25 \mu \mathrm{m})$. Further details are provided in Ref. [41] 
(a)
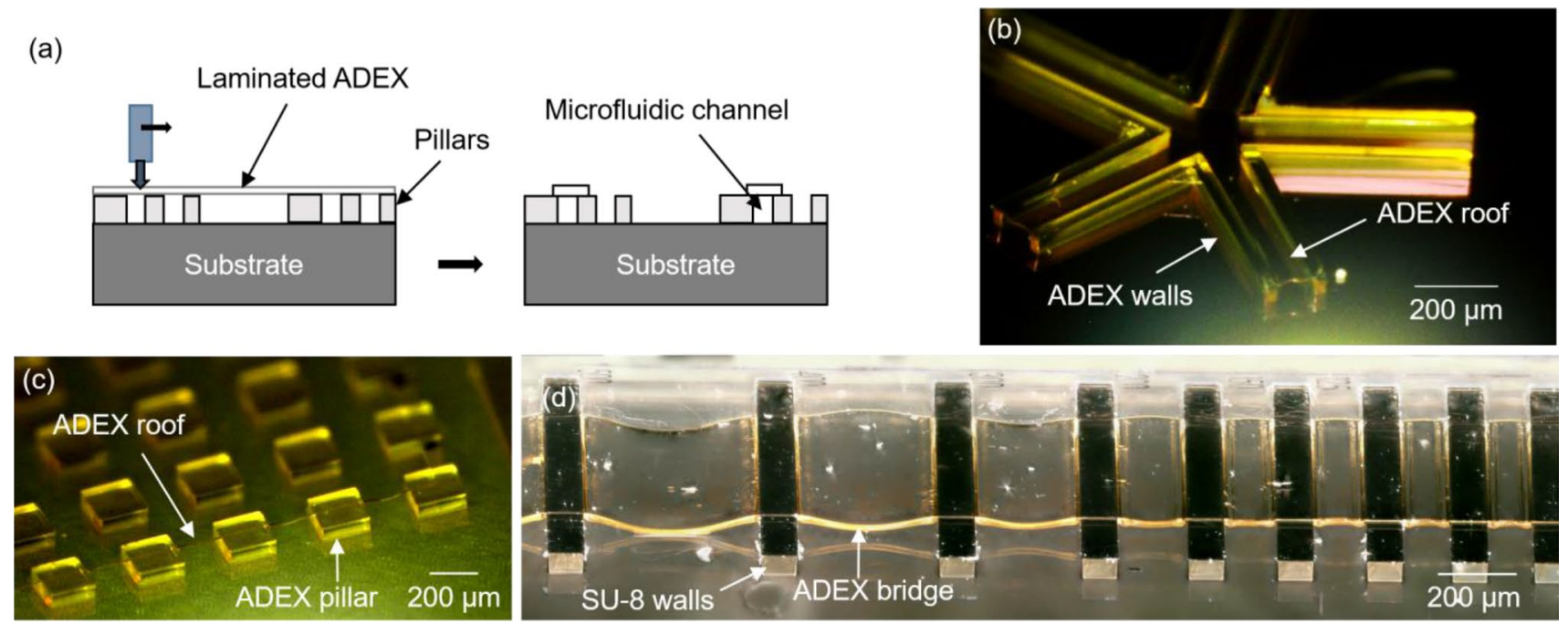

Fig. 4 DFP structures for microfluidic application on silicon oxide: a Scheme of the fabrication of microfluidic channels from laminated DFPs in a multilayer system (impossible with SU-8). b Star-shaped

structures were created with identical parameters, supported by $75 \mu \mathrm{m}$ high $200 \times 200 \mu \mathrm{m}^{2}$ pillars (Fig. $4 \mathrm{c}$ ).

These structures were all manufactured from DFP ADEX as illustrated in Fig. 4a. It is hardly possible to realize such wall/lid-architectures yielding hollow channels and cavities reproducibly with homogeneous resist thickness by employing conventional spin-on resists such as SU-8. The channels would readily fill-up with the liquid photoresist. Beyond the successful proof-of-principle, the examples in Fig. 4 as well provide information about the limitations and persisting challenges of microfluidic fabrication with this strategy. First, the mechanical properties of ADEX DFP set some geometrical limitations for the overall channel design. The critical dimension (CD) of the patterning scale and the aspect ratio depend on the overall utilized lithographic technique as well as on the thickness of the DFP (according to product data sheet: $2 \mu \mathrm{m}$ resolution for $5 \mu \mathrm{m}$ thick DFP ADEX, $7 \mu \mathrm{m}$ resolution for $50 \mu \mathrm{m}$ thickness [48]). Wall thicknesses down to a $\mathrm{CD}$ of $5 \mu \mathrm{m}$ and aspect ratios up to at least 15:1 (Fig. 3b) were realized for channel walls being $75 \mu \mathrm{m}$ in height, which is a higher value for the aspect ratio than the ratio of 50:7 that is stated in the product data sheet [48]. The CD for the lid thickness was in our study merely limited by the thickness of the DFP due to a minimum available ADEX DFP thickness of $5 \mu \mathrm{m}$. Moreover, the bridgeable wall distance sets a critical constraint. Figure $4 \mathrm{~d}$ shows a $100 \mu \mathrm{m}$ high SU-8 wall that is covered by a $15 \mu \mathrm{m}$ thick ADEX lid (fabrication parameters analog to Fig. 4b, c). Obviously, the lid is able to span across the distance without any noticeable bending for an intended channel width up to roughly $150 \mu \mathrm{m}$. If the bridged distance exceeds $200 \mu \mathrm{m}$, the lid tends increasingly with growing channel width to microfluidic channels (walls: $75 \mu \mathrm{m}$ ADEX, roof: $5 \mu \mathrm{m}$ ADEX). c ADEX-pillars $(75 \mu \mathrm{m})$ with ADEX-roof $(5 \mu \mathrm{m})$. d Spin-coated SU8 -walls $(100 \mu \mathrm{m})$ with ADEX-roof $(15 \mu \mathrm{m})$

so-called sagging. At a bridged distance of $500 \mu \mathrm{m}$ the maximum lid sagging occurring at its center is equal to the wall high of $100 \mu \mathrm{m}$ for the here utilized $15 \mu \mathrm{m}$ thick ADEX film. As shown in Fig. $4 \mathrm{c}$, the $5 \mu \mathrm{m}$ thick lid bridges a distance of $200 \mu \mathrm{m}$ without any recognizable sign of sagging. Hence, the achieved aspect ratio without recognizable sagging for the lid was about 40:1. As shown and discussed for another DFP-material by Wangler et al. [36], an increased lamination roller gap, i.e. by reduced lamination pressure, and a reduced lamination temperature (possible range for ADEX between 50 and $70{ }^{\circ} \mathrm{C}$ [48]) are potential approaches to reduce the impact of sagging for DFP suspended structures. A clear dependence of the degree of sagging on the ADEX thickness used cannot be derived from the present set of experiments. Overall, the double-layer structures presented in Fig. 4 demonstrate a facile and rapid manufacturing of 3D-structures by means of multiple ADEX-layers but does not set a limit. DFPs represent already a central component in the shown DFP-based microfluidic channel assembly, but there is still the need for a channel carrier substrate like silicon, glass and polymeric foils, such as polyimide $[49,50]$. Therefore, the question arises whether or not these carrier substrates can be exchanged by patterned DFPs.

\subsection{Lamination-Free Implementation of DFPs}

Free-standing polymeric MEMS architectures were so far mainly realized by spin-coating SU- 8 onto a so-called sacrificial layer that is finally removed to release the functional microstructure. This sacrificial layer technique is well established and was, for instance, used as well in the aforementioned reference [11]. Such a sacrificial layer approach can 
in principle also be applied to DFPs. However, introducing a sacrificial layer or substrate increases the process complexity, time-consumption and costs as well as the environmental impact. Hence, we propose here to employ DFPs directly as flexible and photostructurable substrate materials that makes further substrate materials and sacrificial layers obsolete.

In this regard, as-purchased DFPs comprising ADEX and SUEX were directly exposed via UV laser lithography without any lamination to a substrate; but the DFPs were still attached to their respective bottom PET liner (scheme Fig. 5a). Detailed studies of the fabrication parameters for both materials are attached in the supplementary materials in Sect. 3. Figure 5e, f show an exemplary polymeric grid test structure manufactured in this manner from $75 \mu \mathrm{m}$ thick ADEX and $250 \mu \mathrm{m}$ thick SUEX, respectively. This demonstrates that direct lithography is possible with ADEX and SUEX DFP. It is remarkable that even the thickest available SUEX-DFP with $250 \mu \mathrm{m}$ thickness can be exposed in this manner. Figure 5e, f illustrate, furthermore, the optical appearance of these materials after exposure. Both materials keep a certain transparency. In contrast to SUEX that is almost colorless to slightly yellowish, ADEX turns reddish after exposure and development. The structures also remain flexible after exposure (Fig. 5c, d). The implemented
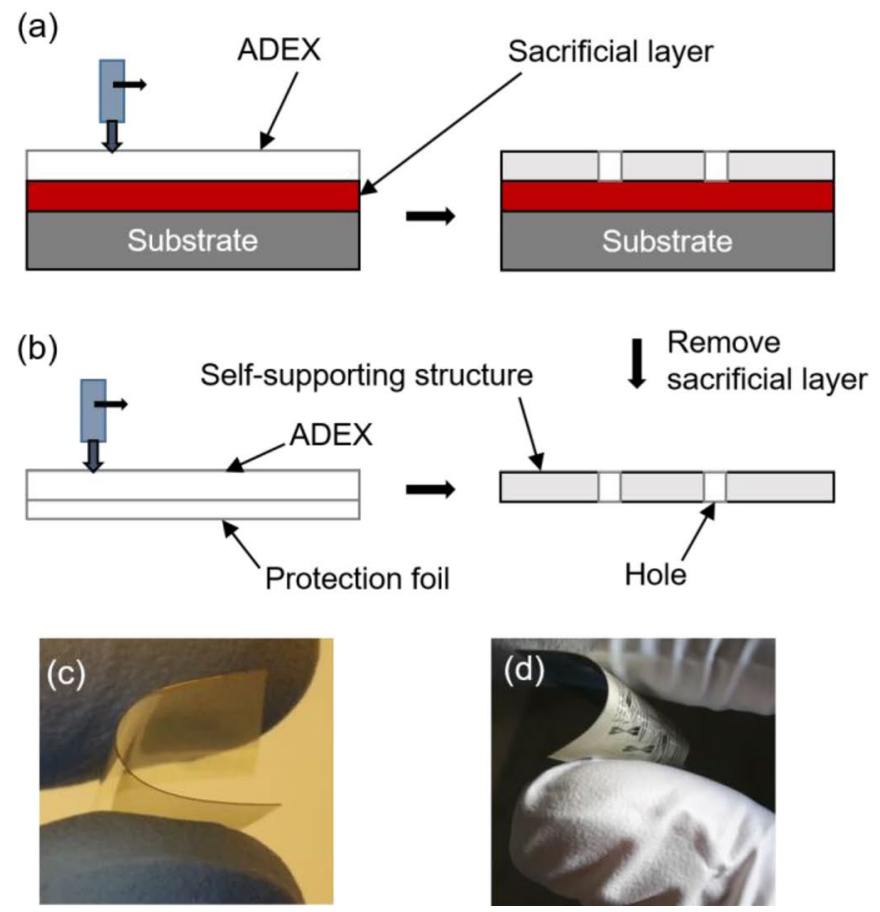

Fig. 5 Polymeric structure microfabricated by exposure of DFPs with laser lithography: a Microfabrication scheme of self-supported polymeric structures made from laminated DFPs on a sacrificial substrate similar to microfabrication with SU-8 [27]. b Microfabrication scheme of self-supported polymeric structures by direct laser lithography on DFPs (impossible with SU-8). c Flexibility of exposed and lithography laser is focused on the resist surface, however, it spreads with increasing thickness from the surface, i.e. deeper into the resist layer. Thicker resists therefore lead to an increased structure width at the bottom surface, compared to the feature top. Also, scattering of the UV-light can increase the size of the exposed patterns with increasing depth in the DFP. These effects are reasons for the resolution limitation in thicker samples and are expected to depend on the DFP material, thickness, and exposure method. For the experiments, ADEX was exposed using a laser power of $3.6 \mathrm{~mW}$, post-exposure baked, and developed.

Another significant benefit that comes along with this microfabrication approach, aside from a reduction of process steps and associated environmental impacts, is the ability to tailor the mechanical compliance of the film. This feature is so far hardly considered. However, it enables further degrees of freedom for future polymer-based MEMS architectures. First, the rigidity can be modified directly by adjusting the hardbake temperature applied after DFP exposure, here in the range of $150-200{ }^{\circ} \mathrm{C}$ for $1-2 \mathrm{~h}$. Second, rigidity can be modified by patterning of the DFP substrate, as shown in Fig. 5, or by means of grayscale lithography, which as well enables to alter the mechanical properties based on the
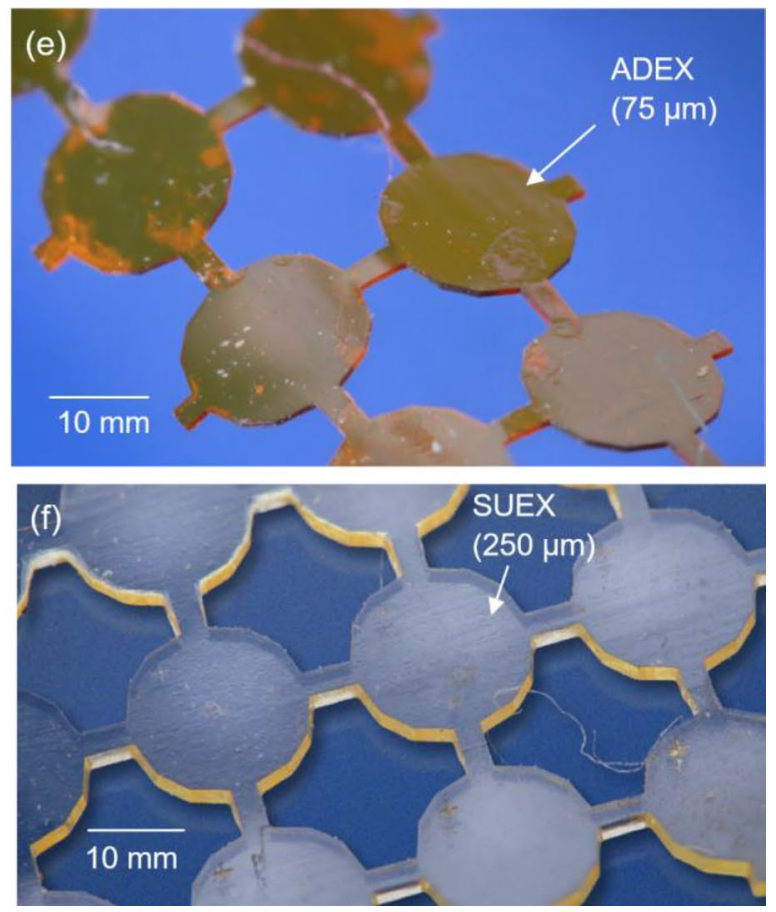

developed $75 \mu \mathrm{m}$-thick ADEX DFP (size: $20 \times 20 \mathrm{~mm}^{2}$ ). d Flexibility of a $75 \mu \mathrm{m}$-thick ADEX DFP with metal structures suggesting the use as a flexible substrate (size: $20 \times 20 \mathrm{~mm}^{2}$ ). e Semi-transparent $75 \mu \mathrm{m}$-thick ADEX DFP showing the characteristic reddish colour. $\mathbf{f}$ Transparent $250 \mu \mathrm{m}$-thick SUEX DFP 
overall morphology (film thickness variation). Mechanical compliance can be therefore even locally adjusted.

\subsection{Lamination-Free DFP Architectures}

Lamination-free exposure of DFPs would in principle allow to create microfluidic channels and to embed various transducers from single devices to the aforementioned polymer mesh electronics [13]. We focus here in this regard to three crucial aspects of microdevice fabrication on top of DFPs, namely: creation of (1) alignment markers for multilevel processes, of (2) metal structures, e.g. for electrical contacts, and of (3) polymeric elements, e.g. to create further passive components or passivation layers. The studies were done for $75 \mu \mathrm{m}$ thick ADEX DFP.

A central aspect of microfabrication are markers that enable precise alignment of patterns within multilevel processes. Alignment markers are frequently created by specifically designed metal deposits that provide a distinct contrast within photolithographic and electron-beam lithography alignment. By employing laser lithography directly on the DFP, we observed that in correlation with high laser power (e.g. $60 \mathrm{~mW}$ ) a burn-in effect can be triggered in the DFP ADEX that changes the color locally from transparent reddish to black (supplementary materials Sect. 4). By exploiting this effect, alignment marker deposition processes were omitted and markers (e.g. letters, numbers, symbols) were directly created together with the exposure of the respective DFP layer.

Figure $6 \mathrm{c}$ exemplifies a marker that was directly burned into ADEX DFP (a detailed parameter study is given in the supplementary materials in Fig. 3a). The exposure energy dose is notably about almost 100 times higher than the dose used for a regular exposure of ADEX. Hence, an according overexposure around the burned-in markers is inevitable. The overexposure must be therefore considered in the overall layout, e.g. by placing markers in sufficient distance (ca. 50-100 $\mu \mathrm{m}$ ) to fine patterns. The CD for the burned-in patterns was about $2 \mu \mathrm{m}$. However, significant size deviations were observed between burned-in patterns of identical dose. This could be, for instance, a result of varying focus accuracy or surface quality, and might limit the reproducibility with respect to the size of small burned-in patterns. However, this should not alter the location of the center of a marker and, hence, not pose an issue for alignment accuracy with symmetrical markers. Compared to metallic markers, the advantages gained by eliminating several process steps are obvious. For example, 81 markers, as shown in Fig. 6c, were created on a sample field of $20 \times 20 \mathrm{~mm}^{2}$ within $5 \mathrm{~min}$. Thus, this approach is a rapid, cheap, and green alternative for a marker generation on DFPs that yield still sub-micrometer alignment accuracy, as systematic tests of manual-optical alignment confirmed.

(a)
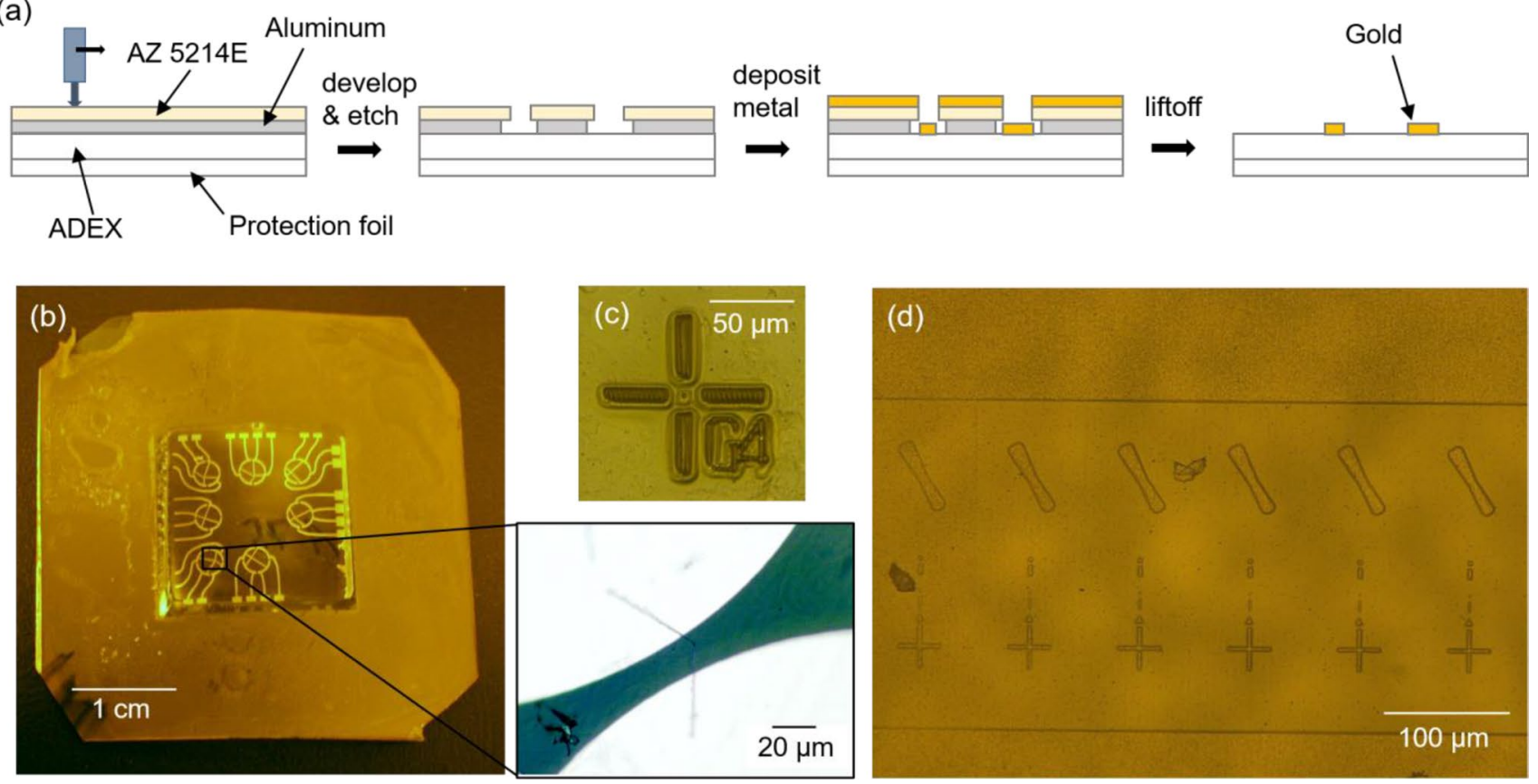

Fig. 6 Lithographic modifications atop a $75 \mu \mathrm{m}$ thick ADEX-DFPsubstrate: a Scheme of metal deposition on ADEX, using a sacrificial aluminum layer as a reflective substrate surface. b $20 \times 20 \mathrm{~mm}^{2}$ gold patterns used as contact metals for the integration of kinked silicon nanowires (see magnified view). c Alignment marker burned in by laser lithography. d Polymeric structures made from SU-8 2000.5 
Metal structures are an indispensable element within miniature systems and are, for instance, frequently required for electrical contacts to operate electrodes, piezo-elements and piezoresistors, capacitors, field-effect transistors, and further components. Considering our lamination-free DFP approach, metal structures must be manufactured after exposure of the DFP, but prior to the post-exposure bake and development if spin-coating resists, that generally require planar substrates without holes or openings, shall be implemented (e.g. for lift-off). Notably, longer storage under yellow light (up to several days) between DFP exposure and post-exposure bake did not deteriorate any of the following processes.

To create metal patterns, similar to the ones shown in Fig. $6 \mathrm{~b}(100 \mathrm{~nm}$ thick gold patterns with $5 \mathrm{~nm}$ titanium as adhesion promotor) on ADEX DFP, the well-known lift-off process for micropatterning was utilized in the following manner that is schematically depicted in Fig. 6a. First, an aluminum layer, nominally $20 \mathrm{~nm}$ thicker than the intended metal patterns, were deposited on ADEX by thermal evaporation, implementing resistive heating rather than electronbeam evaporation. Notably, the overall thermal budget of ADEX must be kept as low as possible (e.g. below $28^{\circ} \mathrm{C}$ ) to avoid structural ADEX deterioration and radiation emitted during electron-beam evaporation can expose ADEX (see supplementary material Sect. 5) and thus, destroy preexposed patterns. The aluminum film serves as a reflective and non-transparent surface finish underneath the lift-off resist, which is here AZ $5214 \mathrm{E}$ (thickness about $1.4 \mu \mathrm{m}$ ). The resist was spin-coated onto the ADEX/aluminum film substrate and patterned again by UV laser lithography. The alkaline resist developer (AZ 726-MIF) enables also direct aluminum patterning during prolonged resist development. Hence, the resist patterns were directly transferred to the aluminum film, which allows metal deposition, here evaporated gold, in the next step directly on ADEX. Alternatively, a 50\% phosphoric acid solution can be used for aluminum patterning as well. Lift-off of the aluminum/resist/gold film was realized in the alkaline developer. This process can be adapted readily to metals that are stable in the alkaline photoresist developer, including gold, silver, platinum, nickel, and copper, but excluding aluminum, chromium, and vanadium [51]. The introduction of a sacrificial aluminum layer and the accompanied processes increase the fabrication complexity. Therefore, top antireflective coatings (AZ Exp. Aquaristi III 45, spin-on, MicroChemicals $\mathrm{GmbH}$ ) were employed in first studies as aluminum replacement between ADEX and AZ 1512 and allowed creation of metal patterns on lamination-free ADEX.

Aside from metal structures, also polymer structures were successfully made on top of ADEX as required, for instance, as structural elements or for the passivation of metal contacts. ADEX itself cannot be applied on unsupported ADEX in combination with lamination so far, since the second DFP-layer would deform the substrate layer and obstruct a subsequent lithographic or thermal treatment. Hence, spinon photoresist SU-8 2000.5 was used. Post-exposure treatment was done together with the DFP substrate, comprising baking at $95{ }^{\circ} \mathrm{C}$ and developing in cyclohexanone for $1 \mathrm{~min}$. Even fine openings of $2 \mu \mathrm{m}$ diameter could be reliably written in a passivation layer with this facile method (see Fig. 6d). More examples for patterned SU-8-structures on DFP ADEX can be found in the appendix.

\subsection{Microprobe Assembly}

Based on the aforementioned lamination-free direct exposure of ADEX DFPs (Fig. 5b), the presented DFP microtechniques and the microprobe examples presented in the introduction, we developed an approach for the exemplary integration of bottom-up grown kinked silicon nanowires (nanofabrication described in the supplementary materials in Section 1) in a liquid-gate field-effect transistor configuration into a microprobe architecture as depicted in Fig. 7.

It is intrinsic to the bottom-up paradigm that nanowires can be grown for instance by the vapor-liquid-solid method [52] in vast numbers. However, for the assembly of nanosensors, only single nanowires are typically required. Hence, several techniques were studied to transfer nanowires from their original growth substrate and to align them on a secondary substrate [53-55]. In this study, as-grown silicon nanowires were released from the growth substrate by ultrasonication and dispersed in double-distilled water. The suspended nanowires were subsequently transferred to the DFP using plain drop-casting onto the substrate in proximity to predefined markers. The alignment markers were directly burned into the DFP and enabled to register the position of nanowires by means of computer-aided image analysis. Based on plain drop-casting, the nanowires are randomly distributed on the DFP, which is a similar case for many transferred nanostructures comprising also graphene and other 2D nanoflakes [56, 57]. Therefore, the functional architecture, such as electrodes and passivation layers must be adopted to the randomly positioned nanowires of interest.

After nanowire deposition and position registration, the DFP was patterned by UV laser lithography such that selected nanowires are situated at the very end of a prospective probe tip, followed by contact metal deposition, by lift-off, and by contact passivation with patterned SU-8 resist. The individual probes were furthermore embedded into a simultaneously patterned DFP net to ease the overall handling. To facilitate the design alignment with respect to the nanowires, we developed software tools (previously published in [58]) that enabled computer-aided read-in of the alignment marker positions combined with a semi-automatic device design positioning. Based on these software 

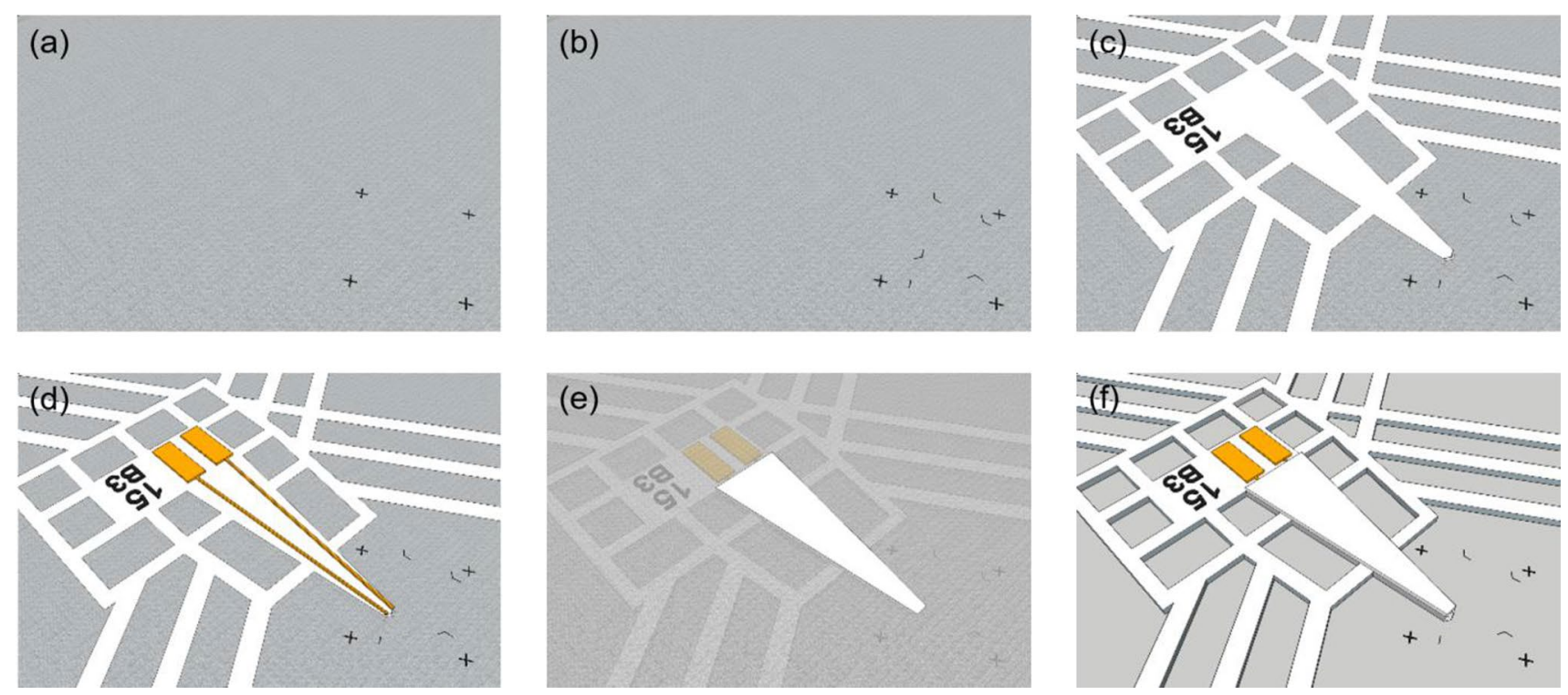

Fig. 7 Schematic illustration of the microprobe assembly process using kinked silicon nanowires as charge transducers in a field-effect transistor configuration: a Photosensitive polymeric DFP ADEX with position markers directly burned into the material with a dose of $60 \mathrm{~mW}$. b Randomly deposited kinked silicon nanowires near the position markers. c Exposure of the polymeric substrate, shaping the tip-shaped sensor as well as the network that stabilizes connects the sensors. d Manufacturing metal contacts in a lift-off-process. e Passivation of metal contacts with an exposed SU-8-coating. f Development of ADEX-substrate together with the SU-8 passivation layer tools, we achieved an alignment accuracy below $1 \mu \mathrm{m}$, confirmed in multilayer alignment tests. Finally, the bottom DFP PET liner was removed and the DFP substrate and the SU8-passivation were simultaneously post-exposure baked and developed. However, notice that this step is challenging due to significantly different development times between SU-8 2000.5 (ca. $1 \mathrm{~min}$ ) and ADEX 75 (ca. $20 \mathrm{~min}$ ). Figure 7 provides a schematic summary of all process steps that represent direct implementations of the aforementioned DFP techniques.

The resulting microprobes and some fabrication stages are shown in Fig. 8. Single probes can be obtained simply by manual knife cutting of the net support structure.

The probe functionality was successfully tested in first experiments by measuring the nanowire resistance as shown in Fig. 8d. The observed symmetrical non-linear current-voltage characteristics should be due to a Schottky barrier at the contact junction [59] originating from the metal-semiconductor contact between the gold metal contacts and the n-doped silicon nanowire.

\subsection{Comparison with SU-8-Based Platforms}

As mentioned before, Qing et al. [11] already showed a strategy for the integration of silicon nanowires based on spin-coated SU-8 as a polymeric substrate. Similar to the approach in Fig. 5a, they employed a sacrificial nickel layer and an SU-8 resist on top of a silicon wafer. After creating the intended architecture, including the metal contacts, and a passivation layer, this sacrificial layer was completely removed in $\mathrm{FeCl}_{3} / \mathrm{HCl}$ to create a released, self-supporting polymer structure [11]. A similar strategy was employed to create the aforementioned injectable mesh electronics [13].

Compared to these strategies, the DFP-based approach presented here reduces the number of required material layers by over 50\%. A detailed comparison is shown in Table 1. The most significant achievements come from the elimination of a carrier substrate, the replacement of metallic markers, and the elimination of the sacrificial nickel layer, which improves the overall bio-compatibility by avoiding cytotoxic effects of nickel [60]. Even though nickel should be removed during the fabrication, remaining traces could be still an issue for biological samples. Similar to SU-8 [61], also DFP ADEX contains potentially cytotoxic antimony compounds. Previous investigation for the likewise epoxy-based SU-8, that showed only minimal leaching in PBS [61], should be transferable to ADEX due to the similar or lower content of antimony compounds below 5\% [62]. Potential issues with these compounds could be, furthermore, avoided by using antimony-free DFPs like SUEX.

Alternative strategies for the release of SU-8 patterns from their substrate based on spin-on photoresists from the product family $\mathrm{AZ}^{\circledR}$ (MicroChemicals $\mathrm{GmbH}$ ) that has an alkaline developer, as a sacrificial layer [63] or fluorocarbon coatings as "non-sticky" surfaces $[64,65]$ each suffer from severe shortcomings. Implementation of AZ-photoresists, on the one hand, sets limitations for further patterning with AZresists atop the SU-8 structure, as required for the creation 

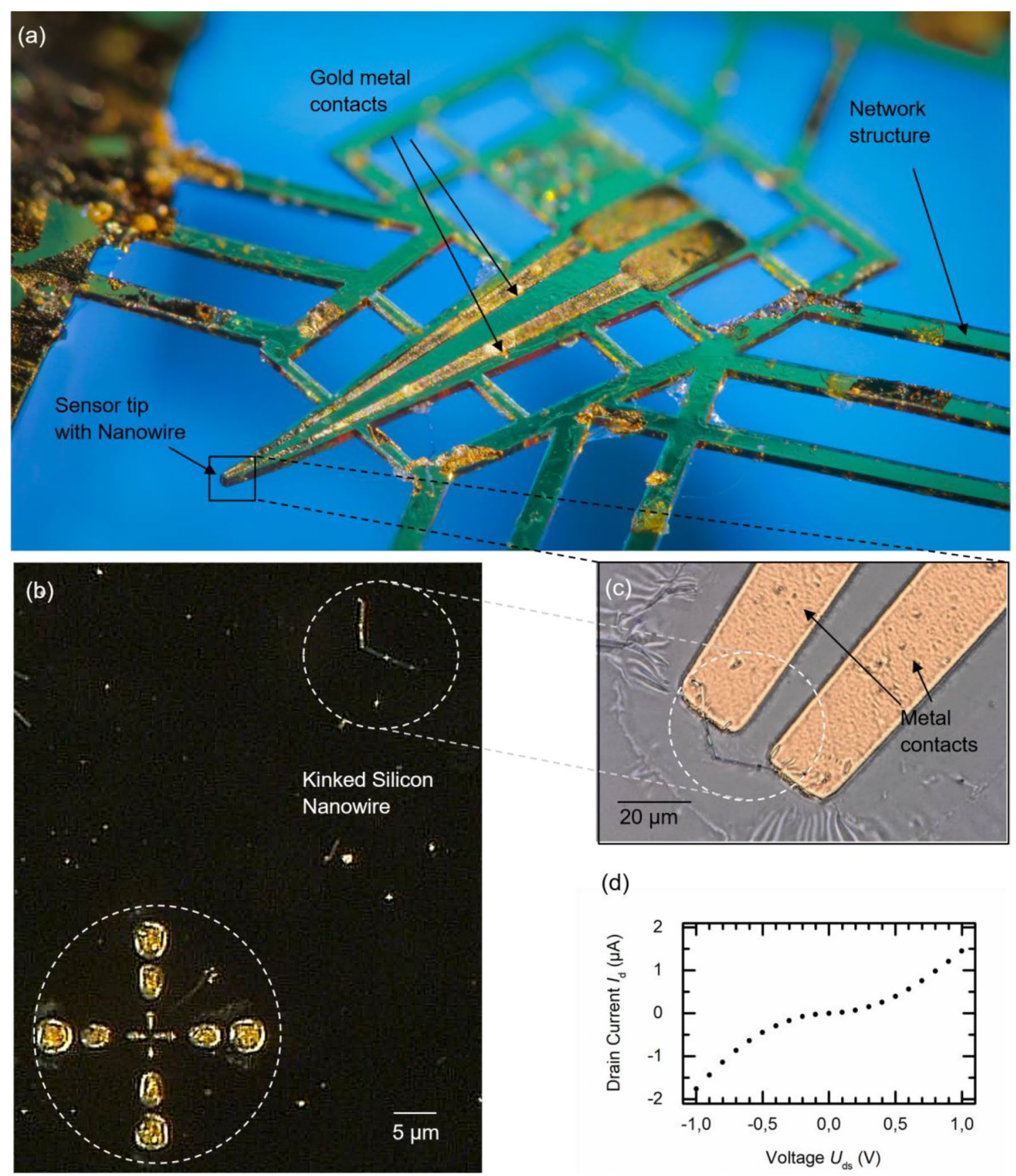

(d)

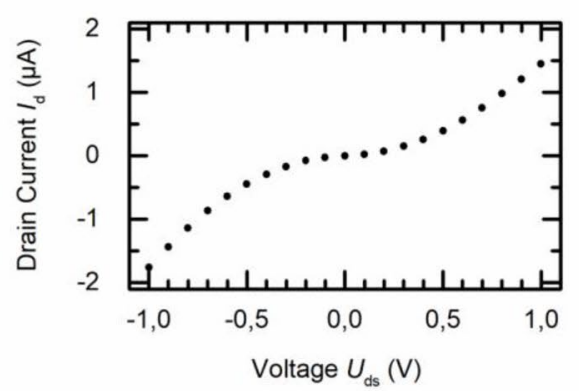

Fig. 8 Electrical integration of silicon nanowires into microprobes: a A representative microprobe made from ADEX DFP. The lift-off quality can be still further improved and is mainly obstructed by the aforementioned issue of inadequate temperature control during thermal evaporation. b Initial ADEX substrate with alignment marker

of metal structures. The release of SU-8-structures from fluoropolymers by mechanical forces, on the other hand, is always a tradeoff between the adhesion quality and the yield of the final devices [65]. Another process-related advantage of using DFPs is their homogeneous film thickness. Due to burned in via laser lithography and a transferred kinked silicon nanowire. c Integrated kinked silicon nanowire with metal contacts. d Electrical resistance measurement of a silicon nanowire integrated on a $75 \mu \mathrm{m}$ ADEX DFP, indicating a Schottky-contact behavior

the high viscosity of SU-8 resists, it is challenging to achieve a homogeneously thick layer via spin-coating.

It is finally worth mentioning again that the presented DFP strategies, mainly for ADEX, are in principle transferable to many other DFPs and naturally to other microsystem designs, too. We believe that we can inspire other 
Table 1 Comparison of two processes for polymeric microprobe fabrication based on spin-on photoresist SU-8 as a substrate [11] and on DFP ADEX, listing here the required material layers

\begin{tabular}{lll}
\hline & SU-8-substrate [11] & ADEX-substrate \\
\hline 1 & $\begin{array}{l}\text { Si-substrate with } \mathrm{SiO}_{2} \text {-layer } \\
2\end{array}$ & $\begin{array}{l}\text { ADEX bottom-plate } \\
\text { connects }\end{array}$ \\
& SU-8 layer & Silicon nanowires \\
3 & Metal contact pads & SU-8-passivation \\
4 & SU-8 layer & \\
5 & Silicon nanowires & \\
6 & SU-8 layer & \\
7 & Thick low-stress SU-8 layer & \\
8 & Metal contacts & \\
9 & LOR 3A and S1805 & \\
10 & &
\end{tabular}

researchers to study DFPs further within the frame of green microfabrication.

\section{Conclusion}

The dry film photoresist (DFP) ADEX as well as partly SUEX DFP were studied to be utilized for a rapid and green microassembly of prospective biomedical microdevices. As a polymeric DFP photoresist, ADEX can be structured by UV lithography without using harsh, toxic, and highly climate-active etchants, such as hydrogen fluoride and sulfur hexafluoride that are typically used for conventional silicon and glass patterning. We demonstrated three exemplary microfabrication approaches with ADEX DFP. First, the DFP-based post-modification of freestanding silicon nitride microcantilevers by dry etching, by localized metal deposition and by creation of functional polymeric structures. Second, the facile assembly of microfluidic channels. Third, the lamination-free use of ADEX via direct exposure by laser lithography. In this regard, we demonstrated the direct burnin of alignment markers for multilevel lithography, the deposition of metal structures by using a lift-off approach, and the creation of polymeric patterns on top of the DFP from SU-8. Finally, we exemplified a device fabrication based on the assembly of an ADEX-based microprobe with an embedded kinked silicon nanowire in field-effect transistor configuration. Similar probes might be usable for instance for future in-vitro cell studies. The presented DFP-based microfabrication strategies are in principle transferable to other DFP systems and device designs and, thus, foster the microdevice assembly in this diverse field that moves on towards a greener microfabrication.

Supplementary Information The online version contains supplementary material available at https://doi.org/10.1007/s40684-021-00367-y.
Acknowledgements We gratefully acknowledge the experimental support by S. Jenisch, T. Huffert, A. Schreiber, A. Minkow (Ulm University), S.N. Mohammad, M. Colditz, and C. Richard (IFW Dresden). Photos in Figs. 5e, f, 8a taken by H. Grandel (Ulm).

Funding Open Access funding enabled and organized by Projekt DEAL. We highly acknowledge funding of this research by the Deutsche Forschungsgemeinschaft (DFG Grant No. GRK2203 PULMOSENS), by the Bundesministerium für Bildung und Forschung (BMBF FKZ: 13N12545), and within the EFRE InfraPro project "ChAMP" (AN: 100330389).

\section{Declarations}

Conflict of interest On behalf of all authors, the corresponding author states that there is no conflict of interest.

Open Access This article is licensed under a Creative Commons Attribution 4.0 International License, which permits use, sharing, adaptation, distribution and reproduction in any medium or format, as long as you give appropriate credit to the original author(s) and the source, provide a link to the Creative Commons licence, and indicate if changes were made. The images or other third party material in this article are included in the article's Creative Commons licence, unless indicated otherwise in a credit line to the material. If material is not included in the article's Creative Commons licence and your intended use is not permitted by statutory regulation or exceeds the permitted use, you will need to obtain permission directly from the copyright holder. To view a copy of this licence, visit http://creativecommons.org/licenses/by/4.0/.

\section{References}

1. Mathur, L., Ballinger, M., Utharala, R., \& Merten, C. A. (2020). Microfluidics as an enabling technology for personalized cancer therapy. Small (Weinheim an der Bergstrasse, Germany), 16(9), e1904321. https://doi.org/10.1002/smll.201904321

2. Iwasaki, Y., \& Seyama, M. (2015). MicroTAS for biosensors. NTT Technical Review, 13

3. Castiaux, A. D., Spence, D. M., \& Martin, R. S. (2019). Review of 3D cell culture with analysis in microfluidic systems. Analytical Methods, 11(33), 4220-4232. https://doi.org/10.1039/c9ay01328h

4. Shinde, P., Mohan, L., Kumar, A., Dey, K., Maddi, A., Patananan, A. N., et al. (2018). Current trends of microfluidic single-cell technologies. International Journal of Molecular Sciences. https://doi. org/10.3390/ijms19103143

5. Ma, Y.-H.V., Middleton, K., You, L., \& Sun, Y. (2018). A review of microfluidic approaches for investigating cancer extravasation during metastasis. Microsyst \& Nanoengineering. https://doi.org/ 10.1038/micronano.2017.104

6. Chen, C.-C., Zhou, Y., \& Baker, L. A. (2012). Scanning ion conductance microscopy. Annual Review of Analytical Chemistry, 5, 207-228. https://doi.org/10.1146/annur ev-anchem-062011-143203

7. Odijk, M., van der Wouden, E. J., Olthuis, W., Ferrari, M. D., Tolner, E. A., van den Maagdenberg, A. M. J. M., et al. (2015). Microfabricated solid-state ion-selective electrode probe for measuring potassium in the living rodent brain: Compatibility with DC-EEG recordings to study spreading depression. Sensors and Actuators B: Chemical, 207, 945-953. https://doi.org/10.1016/j. snb.2014.06.138 
8. Hollenhorst, M. I., Richter, K., \& Fronius, M. (2011). Ion transport by pulmonary epithelia. Journal of Biomedicine and Biotechnology, 2011, 1-16. https://doi.org/10.1155/2011/174306

9. Wittekindt, O. H., \& Dietl, P. (2019). Aquaporins in the lung. Pflugers Archiv. European Journal of Physiology, 471(4), 519532. https://doi.org/10.1007/s00424-018-2232-y

10. Tian, B., Cohen-Karni, T., Qing, Q., Duan, X., Xie, P., \& Lieber, C. M. (2010). Three-dimensional, flexible nanoscale field-effect transistors as localized bioprobes. Science, 329(5993), 830. https://doi.org/10.1126/science.1192033

11. Qing, Q., Jiang, Z., Xu, L., Gao, R., Mai, L., \& Lieber, C. M. (2014). Free-standing kinked nanowire transistor probes for targeted intracellular recording in three dimensions. Nature Nanotechnology, 9, 142-147. https://doi.org/10.1038/nnano.2013.273

12. Gao, R., Strehle, S., Tian, B., Cohen-Karni, T., Xie, P., Duan, X., et al. (2012). Outside looking nanotube transistor intracellular sensors. Nano Letters, 12, 3329-3333. https://doi.org/10.1021/ nl301623p

13. Liu, J., Fu, T. M., Cheng, Z., Hong, G., Zhou, T., Jin, L., et al. (2015). Syringe-injectable electronics. Nature Nanotechnology, 10(7), 629-636. https://doi.org/10.1038/nnano.2015.115

14. Schuhmann, T. G., Zhou, T., Hong, G., Lee, J. M., Fu, T.-M., Park, H.-G., et al. (2018). Syringe-injectable mesh electronics for stable chronic rodent electrophysiology. Journal of Visualized Experiments. https://doi.org/10.3791/58003

15. Lee, J. M., Hong, G., Lin, D., Schuhmann, T. G., Jr., Sullivan, A. T., Viveros, R. D., et al. (2019). Nanoenabled direct contact interfacing of syringe-injectable mesh electronics. Nano Letters, 19(8), 5818-5826. https://doi.org/10.1021/acs.nanolett.9b03019

16. Jacobs, P., \& Akpinar, I. (2018). Single-use medical devices: Economic issues. Heart Asia, 10(2), e011034. https://doi.org/10.1136/ heartasia-2018-011034

17. Silverio, V., \& Cardoso de Freitas, S. (2018). Microfabrication techniques for microfluidic devices. In Complex fluid-flows in microfluidics (pp. 25-51). Springer. https://doi.org/10.1007/ 978-3-319-59593-1_2

18. Myhre, G., \& Shindell, D. (2014). Anthropogenic and natural radiative forcing. In C. Intergovernmental Panel on Climate (Ed.), Climate change 2013-the physical science basis: Working Group I contribution to the Fifth assessment report of the intergovernmental panel on climate change (pp. 659-740). Cambridge University Press

19. Strobel, C., Sandner, T., \& Strehle, S. (2018). Resistive switching memory based on silver-doped chitosan thin films. MRS Advances, 3(33), 1943-1948. https://doi.org/10.1557/adv.2018.72

20. Zhang, W., Lei, Y., Ming, F., Jiang, Q., Costa, P., \& Alshareef, H. (2018). Lignin laser lithography: A direct-write method for fabricating 3D graphene electrodes for microsupercapacitors. Advanced Energy Materials, 8, 1801840. https://doi.org/10.1002/ aenm. 201801840

21. Lausecker, R., Bollgruen, P., Gleißner, U., \& Wallrabe, U. (2017). Digital lithography based on renewable materials as a tool for environmentally benign microfabrication. Journal of Cleaner Production. https://doi.org/10.1016/j.jclepro.2017.11.102

22. Tsioris, K. (2012). Silk material modification and microfabrication strategies for applications in biosensors and drug delivery. Tufts University

23. Convery, N., \& Gadegaard, N. (2019). 30 years of microfluidics. Micro and Nano Engineering, 2, 76-91. https://doi.org/10.1016/j. mne.2019.01.003

24. Gale, B., Jafek, A., Lambert, C., Goenner, B., Moghimifam, H., Nze, U., et al. (2018). A Review of current methods in microfluidic device fabrication and future commercialization prospects. Inventions. https://doi.org/10.3390/inventions3030060

25. Sharma, A., Mondal, S., Mondal, A. K., Baksi, S., Patel, R. K., Chu, W.-S., et al. (2017). 3D printing: It's microfluidic functions and environmental impacts. International Journal of Precision Engineering and Manufacturing-Green Technology, 4(3), 323334. https://doi.org/10.1007/s40684-017-0038-6

26. Sugimito, T., \& Takahashi, H. (2020). Multidirectional UV lithography via inclined/rotated mirrors for liquid materials. Applied Physics Express. https://doi.org/10.35848/1882-0786/ab9656

27. Zhang, Y., Han, J. H., Zhu, L., Shannon, M. A., \& Yeom, J. (2014). Soft lithographic printing and transfer of photosensitive polymers: Facile fabrication of free-standing structures and patterning fragile and unconventional substrates. Journal of Micromechanics and Microengineering. https://doi.org/10.1088/0960$1317 / 24 / 11 / 115019$

28. Ito, T., Kawaguchi, T., Miyoshi, H., Maruyama, K., Kaneko, S., Ohya, S., et al. (2007). Characterization of a microfluidic device fabricated using a photosensitive sheet. Journal of Micromechanics and Microengineering, 17(3), 432-438. https://doi.org/10. 1088/0960-1317/17/3/003

29. Giacomozzi, F., Mulloni, V., Resta, G., \& Margesin, B. (2015). MEMS packaging by using Dry Film resist. Paper presented at the 2015 XVIII AISEM Annual Conference Trento

30. Yeom, J., \& Shannon, M. A. (2010). Detachment lithography of photosensitive polymers: A route to fabricating three-dimensional structures. Advanced Functional Materials, 20(2), 289-295. https://doi.org/10.1002/adfm.200900686

31. Kukharenka, E., Farooqui, M. M., Grigore, L., Kraft, M., \& Hollinshead, N. (2003). Electroplating moulds using dry film thick negative photoresist. Journal of Micromechanics and Microengineering, 13(4), S67-S74. https://doi.org/10.1088/0960-1317/13/4/ 311

32. Johnson, D. W., Goettert, J., Singh, V., \& Yemane, D. (2011). SUEX dry film resist - a new material for high aspect ratio lithography. Proceedings of SPIE-The International Society for Optical Engineering. https://doi.org/10.1117/12.882872

33. Johnson, D., Goettert, J., Singh, V., \& Yemane, D. (2012). SUEX for high aspect ratio micro-nanofluidic applications. Technical Proceedings of the 2012 NSTI Nanotechnology Conference and Expo, 2, 404-407.

34. Lemke, S., Goettert, P., Rudolph, I., Goettert, J., \& Löchel, B. (2011). Negative resists for ultra-tall, high aspect ratio microstructures. In Proceedings MNE Conference

35. Huesgen, T., Lenk, G., Albrecht, B., Vulto, P., Lemke, T., \& Woias, P. (2010). Optimization and characterization of waferlevel adhesive bonding with patterned dry-film photoresist for 3D MEMS integration. Sensors and Actuators A: Physical, 162(1), 137-144. https://doi.org/10.1016/j.sna.2010.06.008

36. Wangler, N., Gutzweiler, L., Kalkandjiev, K., Müller, C., Mayenfels, F., Reinecke, H., et al. (2011). High-resolution permanent photoresist laminate TMMF for sealed microfluidic structures in biological applications. Journal of Micromechanics and Microengineering, 21(9), 095009. https://doi.org/10.1088/0960-1317/ 21/9/095009

37. Courson, R., Cargou, S., Conedera, V., Fouet, M., Blatche, M. C., Serpentini, C. L., et al. (2014). Low-cost multilevel microchannel lab on chip: DF-1000 series dry film photoresist as a promising enabler. RSC Advances, 4, 54847-54853. https://doi.org/10.1039/ c4ra09097g

38. Meier, R. C., Badilita, V., Brunne, J., Wallrabe, U., \& Korvink, J. G. (2011). Complex three-dimensional high aspect ratio microfluidic network manufactured in combined PerMX dry-resist and SU-8 technology. Biomicrofluidics, 5, 1-10. https://doi.org/10. $1063 / 1.3613668$

39. Kalkandjiev, K. (2015). Microfluidics based on silicon/polymer and all-polymer technologies as an alternative to silicon/glass: A case study for TopSpot printheads. Albert-Ludwigs-Universität Freiburg 
40. Xia, Y., \& Whitesides, G. M. (1998). Soft lithography. Annual Review of Materials Science, 28(1), 153-184. https://doi.org/10. 1146/annurev.matsci.28.1.153

41. Nilsen, M., Port, F., Roos, M., Gottschalk, K., \& Strehle, S. (2018). Facile modification of freestanding silicon nitride microcantilever beams by dry film photoresist lithography. Journal of Micromechanics and Microengineering. https://doi.org/10.1088/ 1361-6439/aaf7e3

42. Cohen, S. R., \& Bitler, A. (2008). Use of AFM in bio-related systems. Current Opinion in Colloid \& Interface Science, 13(5), 316-325. https://doi.org/10.1016/j.cocis.2008.02.002

43. Steffens, C., Leite, F. L., Bueno, C. C., Manzoli, A., \& Herrmann, P. S. (2012). Atomic force microscopy as a tool applied to nano/ biosensors. Sensors (Basel), 12(6), 8278-8300. https://doi.org/10. 3390/s 120608278

44. Cohen-Karni, T., Casanova, D., Cahoon, J. F., Qing, Q., Bell, D. C., \& Lieber, C. M. (2012). Synthetically encoded ultrashortchannel nanowire transistors for fast, pointlike cellular signal detection. Nano Letters, 12(5), 2639-2644. https://doi.org/10. 1021/n13011337

45. Sreenivas, V., Winkler, A., Harazim, S., \& Schmidt, O. (2018). Ultraviolet transmittance of SU-8 photoresist and its importance in multi-wavelength photolithography. Journal of Vacuum Science \& Technology B, 36, 051601. https://doi.org/10.1116/1.5033996

46. James, M. D., David, K. P., Jun, W., Dan, S., Jimmy, T. K. T., Chinheng, C., et al. (2007). Creation of embedded structures in SU-8. Proceedings of SPIE. https://doi.org/10.1117/12.702876

47. Nilsen, M., Dannberg, O., Fröhlich, T., \& Strehle, S. (2020). Direct polymer microcantilever fabrication from free-standing dry film photoresists. Journal of Micromechanics and Microengineering. https://doi.org/10.1088/1361-6439/ab9e4c

48. DJ MicroLaminates Inc. (2016). ADEX epoxy thin film rolls/ sheets-Preliminary Data Sheet.

49. Taptimthong, P., Rittinger, J., Wurz, M. C., \& Rissing, L. (2014). Flexible magnetic writing/reading system: Polyimide film as flexible substrate. Procedia Technology, 15, 230-237. https://doi.org/10.1016/j.protcy.2014.09.076

50. Acton, Q. A. (2012). Issues in nanotechnology and microtechnology: engineering, fabrication, and structural research: 2011 Edition: ScholarlyEditions

51. Williams, K. R., Gupta, K., \& Wasilik, M. (2003). Etch rates for micromachining processing-Part II. Journal of Microelectromechanical Systems, 12(6), 761-778. https://doi.org/10.1109/ JMEMS.2003.820936

52. Schmidt, V., Wittemann, J. V., Senz, S., \& Gósele, U. (2009). Silicon nanowires: A review on aspects of their growth and their electrical properties. Advanced Materials, 21, 2681-2702. https://doi.org/10.1002/adma.200803754

53. Hazut, O., Agarwala, A., Subramani, T., Waichman, S., \& Yerushalmi, R. (2013). Monolayer contact doping of silicon surfaces and nanowires using organophosphorus compounds. JoVE. https://doi.org/10.3791/50770

54. Lee, C. H., Kim, D. R., \& Zheng, X. (2010). Orientation-controlled alignment of axially modulated pn silicon nanowires. Nano Letters, 10(12), 5116-5122. https://doi.org/10.1021/nl103 630c

55. Roßkopf, D., \& Strehle, S. (2016). Surface-controlled contact printing for nanowire device fabrication on a large scale. Nanotechnology, 27, 8. https://doi.org/10.1088/0957-4484/27/18/ 185301
56. Skippins, A. (2015). Deposition and analysis of graphene thin films. Swansea University

57. He, H., Berglund, S. P., Rettie, A. J. E., Chemelewski, W. D., Xiao, P., Zhang, Y., et al. (2014). Synthesis of BiVO4 nanoflake array films for photoelectrochemical water oxidation. Journal of Materials Chemistry A, 2(24), 9371-9379. https://doi.org/10. 1039/c4ta00895b

58. Roos, M. M., Huffert, T., Puchinger, A., \& Strehle, S. (2018). Rapid micro-nano-integration of single silicon nanowires in $2 \mathrm{~d}$-sensor arrays using automated software tools. In GMM-Fachbericht 92: Micro-Nano-Integration; 7th GMM-Workshop, 23-27, VDE-Verlag

59. Sze, S. M., \& Ng, K. K. (2007). Physics of Semiconductor Devices. Wiley. https://doi.org/10.1002/0470068329

60. Macomber, L., \& Hausinger, R. P. (2011). Mechanisms of nickel toxicity in microorganisms. Metallomics, 3(11), 1153-1162. https://doi.org/10.1039/c1mt00063b

61. Nemani, K. V., Moodie, K. L., Brennick, J. B., Su, A., \& Gimi, B. (2013). In vitro and in vivo evaluation of SU-8 biocompatibility. Materials Science \& Engineering, C: Materials for Biological Applications, 33(7), 4453-4459. https://doi.org/10.1016/j.msec. 2013.07.001

62. Rasmussen, K. H., Keller, S. S., Jensen, F., Jorgensen, A. M., \& Hansen, O. (2013). SU-8 etching in inductively coupled oxygen plasma. Microelectronic Engineering, 112, 35-40. https://doi.org/ 10.1016/j.mee.2013.05.011

63. Lau, K. H., Giridhar, A., Harikrishnan, S., Satyanarayana, N., \& Sinha, S. K. (2013). Releasing high aspect ratio SU-8 microstructures using AZ photoresist as a sacrificial layer on metallized $\mathrm{Si}$ substrates. Microsystem Technologies, 19(11), 1863-1871. https:// doi.org/10.1007/s00542-013-1740-0

64. Haefliger, D., Nordström, M., Rasmussen, P. A., \& Boisen, A. (2005). Dry release of all-polymer structures. Microelectronic Engineering, 78-79, 88-92. https://doi.org/10.1016/j.mee.2004. 12.013

65. Keller, S., Haefliger, D., \& Boisen, A. (2007). Optimized plasmadeposited fluorocarbon coating for dry release and passivation of thin SU-8 cantilevers. Journal of Vacuum Science \& Technology B: Microelectronics and Nanometer Structures. https://doi.org/10. $1116 / 1.2806960$

Publisher's Note Springer Nature remains neutral with regard to jurisdictional claims in published maps and institutional affiliations.

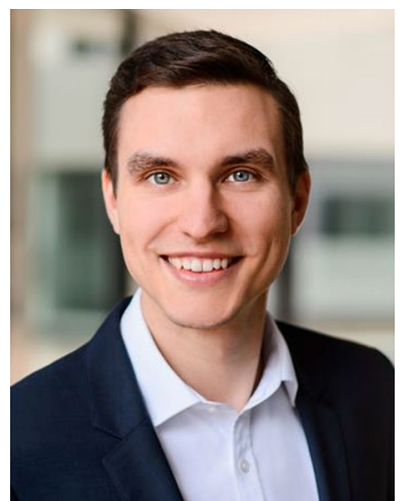

Michael M. Roos received his B.Sc. and his M.Sc. in chemical engineering at Technical University of Munich, Germany. He joined the interdisciplinary graduate school PULMOSENS at Ulm University as a PhD candidate at the Institute of Electronic Devices and Circuits. At the boundary between chemistry, engineering, medicine, and IT, his research is dedicated to realize new types of miniature MEMS-devices and biochemical 
sensors based on electrochemical and semiconductive measurement principles.

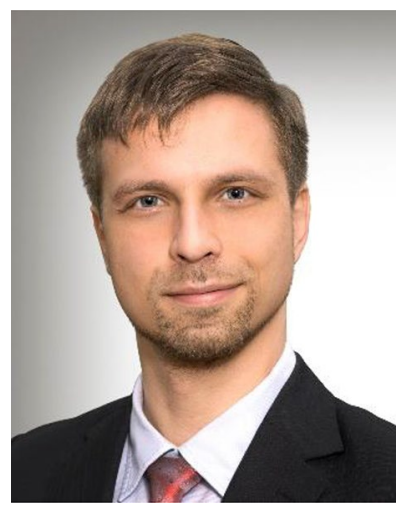

Dr.-Ing. Andreas Winkler holds a $\mathrm{PhD}$ in Material Sciences and a Diplom in Bioprocess Engineering from Technical University Dresden, Germany as well as a Diplom in Microsystems Technology from the University of Applied Sciences Mittweida, Germany. He is scientific fellow of the Leibniz IFW Dresden, Germany and member of the SAWLab Saxony - the competence and application center for acoustoelectronic fundamentals, technologies and devices. His research interests concern the investigation of improved material systems for integrated sensor and actuator devices, especially in the field of microacoustics and emerging applications. His field of work also comprises material and technology related problems, including the increase of power durability of interdigital transducers, the development of new manufacturing methods in the field of acoustofluidics.

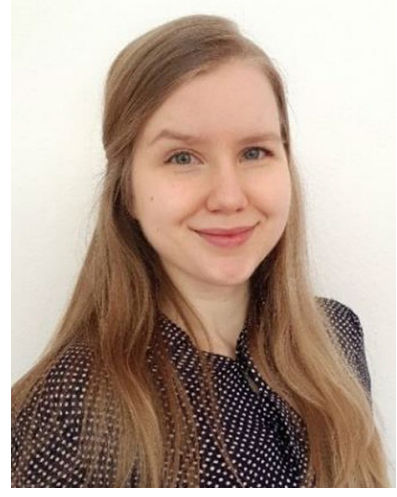

Madeleine Nilsen received her B.Sc. in materials science and nanotechnology from the University of Oslo, and her M.Sc. from Ulm University. She is currently a $\mathrm{PhD}$ candidate at the Institute of Electronic Devices and Circuits at Ulm University, focusing on micro/nanofabrication of cantilever-based systems towards (bio-)chemical sensor applications. Her main research demonstrates the capabilities of high-throughput, dry film photoresist lithography to both, create sensitive polymeric microcantilever probes, as well as to reliably pattern silicon-based microstructures.

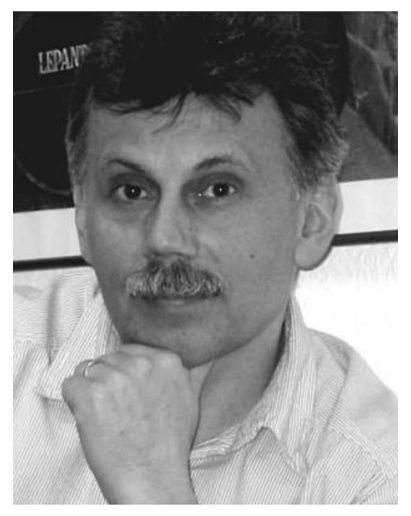

Siegfried Menzel received a diploma in Electrical Engineering and his doctoral degree in Material Science at the University of Technology Dresden, Germany. He worked on plasma assisted thin film processes and low temperature plasma physics. In 1990 he moved to the Leibniz IFW Dresden where he focused on laser material processing and solid state analysis. Now he is group leader of the SAWLab of the IKM of the IFW and coleader of the competence and application center for acoustoelectronics (SAWLab-Saxony). His scientific interests include advanced thin films and thin film analysis, SAW/BAW technology and related processes, transport phenomena in metallizations as well future applications of SAW devices.

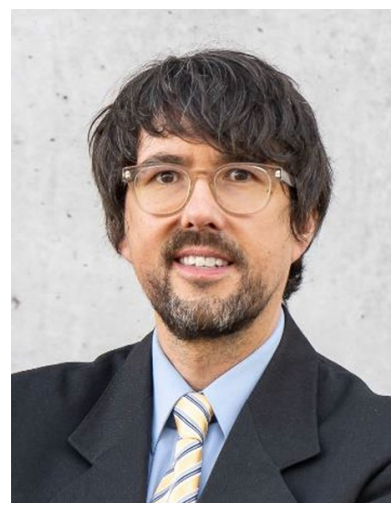

Steffen Strehle received his doctorate for his research in the field of interconnect materials for integrated circuits at TU Dresden (Germany). He worked as a scientist at the IFW Dresden e.V. (Germany) and later at the Institute for Semiconductor and Microsystems Technology at TU Dresden. At the beginning of 2010, he moved to Harvard University (USA) where he focused on silicon nanowire synthesis and the microtechnological assembly of nanowire-based sensors. Later, he had a professorship in the field of semiconductor systems at Ulm University (Germany). Beginning of 2019, he moved to the Technische Universität Ilmenau (Germany), where he heads since then the Microsystems Technology group. (Image: S. Günther-Müller) 